\title{
A Multidimensional Assessment of Successful Aging Among Older People Living with HIV in Palm Springs, California
}

\author{
Logan Z. Marg, ${ }^{1}$ Omeid Heidari, ${ }^{2}$ Jeff Taylor, ${ }^{3}$ Chuck Marbley, ${ }^{4}$ Steve Scheibel, ${ }^{4,}{ }^{\star}$ Rod Hagan, ${ }^{4}$ \\ Ilhem Messaoudi, ${ }^{5}$ Norma Mendoza, ${ }^{5}$ and Brandon Brown ${ }^{6}$
}

\begin{abstract}
We assessed successful aging among older people living with HIV (PLWH) compared with older people without HIV. One hundred ten older men and women in Palm Springs, California completed a self-administered 28-question survey, which collected data on physiological and psychosocial factors related to successfully aging with HIV, including demographics, HIV status, sexual activity, health and well-being, experiences of stigma or discrimination, feelings of isolation, receipt of disability benefits, work and volunteer participation, and presence of comorbid infectious diseases, noninfectious diseases, and geriatric syndromes. Most participants were male $(96.4 \%)$, non-Hispanic white $(84.5 \%)$, college educated $(61.7 \%)$, and ranged in age from 55 to 87 years (median $=64$ years). Respondents with HIV were significantly older than those without HIV $(p=.04)$. The overall prevalence of two or more comorbid conditions across the sample was $59.1 \%$. PLWH were more likely to report depression $(p=.008)$. PLWH were also significantly more likely to report having a current sex partner living with HIV $(p<.001)$ and receiving disability benefits than people without HIV $(41.9 \%$ vs. $6.3 \%)$. Among PLWH, there was a significant relationship between not working or volunteering and feelings of isolation $(p=.005)$. For people without HIV, we found a significant relationship between feelings of isolation and not living with someone $(p<.001)$, but there was no such relationship among PLWH-possibly reflecting the strength of the support network for PLWH in Palm Springs. Our findings suggest that older PLWH experience successful aging to a similar degree compared with their peers without HIV. However, depression and social isolation remain highly salient issues that threaten successful aging and with which PLWH must contend.
\end{abstract}

Keywords: HIV, successful aging, Coachella Valley, Palm Springs

\section{Introduction}

A CCORDING TO THE Centers for Disease Control and Prevention, 300,000 people living with HIV (PLWH) are $\geq 55$ years of age. ${ }^{1}$ Advances in antiretroviral therapy (ART) have transformed HIV from a death sentence to a mostly manageable chronic disease, allowing people to live up to 50 years beyond diagnosis. ${ }^{2,3}$ As PLWH age, they face many psychosocial and health challenges, including high rates of multimorbidity, social isolation, stigma, and agism. ${ }^{4-7}$ Given these challenges, the interaction between HIV and the aging process can make successful aging more difficult for PLWH.
There is no standard definition of successful aging, but it is widely recognized as a multidimensional construct that encompasses physical health (e.g., physical impairment, longevity), psychological health (e.g., depression), and social engagement (e.g., involvement in meaningful relationships and/or social activities, social support).$^{8-10}$ While early definitions of successful aging tend to emphasize physiology, such as sustained physical health and the absence of disease and disability, ${ }^{10}$ more recent conceptualizations emphasize the importance of psychosocial components (e.g., social support, mental health). ${ }^{8,9,11}$ Indeed, some argue that social and/or psychological mechanisms can compensate for physiological deficits and limitations,

\footnotetext{
${ }^{1}$ Department of Sociology, University of California, Riverside, Riverside, California

${ }^{2}$ School of Nursing, Johns Hopkins University, Baltimore, Maryland.

${ }^{3}$ HIV+Aging Research Project-Palm Springs, Palm Springs, California.

${ }_{5}^{4}$ Borrego Health, Cathedral City, California.

${ }^{5}$ School of Biological Sciences, University of California, Irvine, Irvine, California.

${ }^{6}$ School of Medicine, University of California, Riverside, Riverside, California.

*Deceased.
} 
suggesting that older individuals with chronic health conditions, including PLWH, can age successfully if those mechanisms are in place (e.g., valued social ties, social activities, etc.). ${ }^{12}$ For example, research shows that depression has a large effect on daily life and is associated with less successful aging. ${ }^{9,13-15}$ Among older PLWH, depression often stems from feelings of isolation, lack of social support, and grief due to the loss of friends and family. ${ }^{9,16-18}$ However, research suggests that depressive symptoms can be reduced through social involvement (e.g., volunteering, working, etc.), which often results in widened social networks and increased social support. ${ }^{19}$

There is a small but growing literature on experiences and definitions of successful aging among older PLWH. Most research suggests that social engagement is critical for older PLWH to achieve successful aging. Supporting Rowe and Kahn's model of successful aging in which a major element is active engagement in life, ${ }^{10}$ research has found that volunteering is closely linked to older PLWH's well-being and their personal experience of successful aging. ${ }^{13,20}$ One study found that volunteering leads to increased self-esteem, a sense of personal fulfillment, and strengthened social networks. ${ }^{20}$ Another study found that while older PLWH had worse self-reported successful aging as well as physical and mental health compared with older people without HIV, the two groups did not differ on key psychosocial aspects related to successful aging, including levels of optimism, resilience, sense of personal mastery, and social support. ${ }^{21}$ In addition, there was a stronger relationship between those psychosocial components and self-reported successful aging than between successful aging and duration or severity of HIV. ${ }^{21}$ Thus, research suggests that despite the numerous challenges associated with aging with HIV, it is possible for older PLWH to age successfully.

Overall, there remains a dearth of research that assesses successful aging among older PLWH, and there are few direct comparisons between older adults living with and without HIV. However, as more PLWH become older, it is increasingly important to understand people's experiences aging with HIV to identify ways to do so successfully. More research is needed to develop tailored interventions that promote successful aging among older PLWH. Thus, this study sought to assess successful aging among older PLWH by comparing older PLWH with older people without HIV on measures of physiological and psychosocial factors, such as physical health and social engagement.

\section{Setting}

Located in Riverside County, California, the Coachella Valley (CV) is home to Palm Springs and one of the oldest populations of PLWH in the United States, partially due to inmigration of PLWH to this retirement community. The average age of PLWH in CV is 51 years compared with 41 in Riverside County. ${ }^{22}$ Moreover, $24 \%$ of PLWH in CV are $>60$, which is over triple the percentage of older PLWH in Riverside County. ${ }^{22}$ Thus, Palm Springs (and CV) is an ideal setting to investigate successful aging among older PLWH.

\section{Materials and Methods}

Participants were recruited in Palm Springs from one of three community-based organizations, a health center, and a grassroots HIV program. A 28-question self-administered paper survey (Appendix A1) collected multidimensional data related to successful aging with HIV, including demographics and information about HIV status, sexual activity, physical health and well-being, experiences of stigma or discrimination, feelings of isolation, receipt of disability benefits, and work or volunteering. Participants also indicated whether they had infectious diseases (hepatitis, herpes, pneumonia, shingles, and other STDs), noninfectious diseases (cancer, dermatologic problems, diabetes, heart condition, hypertension, neurological problems, neuropathy, respiratory problems, migraines, and stroke), and geriatric syndromes (depression, arthritis, vision loss, hearing loss, and broken bones). In addition, participants were prompted to describe experiences of discrimination in an open-ended survey question. All data were self-reported. Inclusion criteria included being $\geq 55$ years of age and reporting overall good general health. The Institutional Review Board of the University of California, Riverside reviewed and approved this study, and the University of California, Irvine obtained a reliance registry. All participants completed written informed consent to participate in this study.

\section{Statistical analysis}

Univariate analysis was performed to describe variable frequencies with stratification by HIV status. Chi-squared or Fisher's exact tests were computed to determine significant differences in the reported frequencies of variables by the two groups. Analyses were conducted using Stata version 14.2.

\section{Results}

A total of 110 participants completed the survey, with age ranging from 55 to 87 years (median $=64$ years). Sixty-two participants were PLWH; the remaining 48 participants were those without HIV. PLWH were significantly older than people without HIV $(p=.04)$. Most participants were men (96\%), non-Hispanic white (84\%), and in a relationship or married (53\%). Most participants (62\%) had a 4-year college degree or higher (Table 1). Compared with those without HIV, PLWH were significantly less likely to report being in a relationship $(p=.04)$.

Most PLWH (95\%) and people without HIV (89\%) rated their overall health as "Excellent," "Very Good," or "Good" $(p=.39)$. There were no differences in pain experienced during activities of daily living (ADLs) $(p=.64)$, and $24 \%$ of all participants reported a pain score $>5$. There was a significant difference in receiving disability benefits between PLWH $(42 \%)$ and those not living with HIV $(6.3 \%)(p<.001)$.

There were no significant differences between PLWH and people without HIV in noninfectious diseases and geriatric syndromes (Fig. 1). However, PLWH were significantly more likely to report one or more infectious diseases than people without HIV, because HIV is itself an infectious disease $(p<.001)$. In addition, PLWH and people without HIV reported a similar frequency of two or more comorbid conditions. The total prevalence of two or more comorbid conditions across the sample was $59.1 \%$. The top comorbidities among the sample included hypertension (14.8\%), neuropathy (12.9\%), arthritis (12.2\%), depression (10.7\%), hearing loss $(9.9 \%)$, vision loss (6.8\%), and diabetes 
Table 1. Demographics and Factors of Successful Aging Among 110 Survey Participants

\begin{tabular}{|c|c|c|c|c|c|c|c|}
\hline & & & & & & & \\
\hline & $\mathrm{n}$ & $\%$ & $\mathrm{n}$ & $\%$ & $\mathrm{n}$ & $\%$ & $\mathrm{p}$ \\
\hline Demographics & & & & & & & \\
\hline $\operatorname{Age}^{\mathrm{a}}$ & 64 & $(6.6)$ & 63 & (6) & 65.5 & $(7.1)$ & .04 \\
\hline Sex & & & & & & & \\
\hline Man & 106 & $(96.4)$ & 60 & $(96.8)$ & 46 & $(95.8)$ & .59 \\
\hline Woman & 4 & $(3.6)$ & 2 & $(3.2)$ & 2 & $(4.2)$ & \\
\hline Race & & & & & & & .82 \\
\hline Non-Hispanic white & 93 & $(84.5)$ & 52 & $(83.9)$ & 41 & $(85.4)$ & \\
\hline Other & 17 & $(15.5)$ & 10 & $(16.1)$ & 7 & $(14.6)$ & \\
\hline Education & & & & & & & .22 \\
\hline High school & 8 & $(7.5)$ & 7 & $(11.5)$ & 1 & $(2.2)$ & \\
\hline Some college & 33 & $(30.8)$ & 21 & (34.4) & 12 & $(26.1)$ & \\
\hline Four-year university & 31 & (29) & 16 & $(26.2)$ & 15 & $(32.6)$ & \\
\hline Graduate school & 35 & $(32.7)$ & 17 & (27.9) & 18 & $(39.1)$ & \\
\hline Relationship status & & & & & & & .04 \\
\hline Single & 45 & $(41.3)$ & 31 & $(50)$ & 12 & $(25.5)$ & \\
\hline In a relationship & 18 & (16.5) & 10 & $(16.1)$ & 8 & (17) & \\
\hline Engaged & 2 & $(1.8)$ & 1 & $(1.6)$ & 1 & $(2.1)$ & \\
\hline Married & 38 & $(34.9)$ & 15 & (24.2) & 23 & $(48.9)$ & \\
\hline Widowed & 6 & $(5.5)$ & 5 & $(8.1)$ & 1 & $(2.1)$ & \\
\hline Factors of successful ag & & & & & & & \\
\hline Felt positive treatment & 41 & $(37.3)$ & 23 & $(37.1)$ & 18 & $(37.5)$ & .96 \\
\hline Felt age discrimination & 33 & $(30)$ & 21 & $(33.9)$ & 12 & $(25)$ & .31 \\
\hline Health rating & & & & & & & .39 \\
\hline Excellent & 16 & $(14.8)$ & 7 & $(11.5)$ & 8 & $(17)$ & \\
\hline Very good & 51 & $(47.2)$ & 29 & $(47.5)$ & 22 & $(46.8)$ & \\
\hline Good & 34 & $(31.5)$ & 22 & $(36.1)$ & 12 & $(25.5)$ & \\
\hline Fair & 6 & $(5.6)$ & 2 & $(3.3)$ & 4 & $(8.5)$ & \\
\hline Poor & 1 & $(0.9)$ & 1 & (1.6) & 0 & (0) & \\
\hline Pain score ${ }^{\mathrm{a}, \mathrm{b}}$ & 2.46 & $(2.4)$ & 2.6 & $(2.3)$ & 2.34 & $(2.4)$ & .64 \\
\hline Exercise days/week ${ }^{\mathrm{a}}$ & 4 & $(2.5)$ & 4 & $(2.5)$ & 3.6 & $(2.4)$ & .41 \\
\hline Receipt of disability & 29 & $(27.6)$ & 26 & (41.9) & 3 & $(6.3)$ & $<.001$ \\
\hline Lived with someone & 59 & $(54.6)$ & 24 & (39.3) & 35 & $(74.5)$ & $<.001$ \\
\hline Pet ownership & 61 & $(56.5)$ & 39 & (63.9) & 22 & $(46.8)$ & .08 \\
\hline Sexually active & 74 & (67.9) & 40 & $(64.5)$ & 34 & $(72.3)$ & .39 \\
\hline HIV+ sex partner & 42 & $(43.7)$ & 34 & $(64.1)$ & 8 & (18.6) & $<.001$ \\
\hline Worked/volunteered & 70 & $(64.8)$ & 41 & $(66.1)$ & 29 & (63) & .74 \\
\hline Felt isolated & 15 & (13.8) & 11 & (17.7) & 4 & $(8.5)$ & .26 \\
\hline
\end{tabular}

Chi-squared or Fisher's exact tests were conducted to determine significant differences between the two groups.

${ }^{a}$ Values reflect $M(S D)$ in first three columns.

${ }^{\mathrm{b}}$ Refers to pain during activities of daily living. Scores are between 1 and 10 .

$\mathrm{SD}$, standard deviation.

(5.3\%). However, PLWH were more likely to report depression $(35.5 \%$ vs. $12.5 \%, p=.008)$.

Overall, most participants $(68 \%)$ reported being sexually active. Among participants who reported knowing the HIV status of their current sex partner, most $(51 \%)$ had a sex partner with HIV and PLWH were more likely to have a sex partner with HIV than people without HIV $(p<.001)$. Less than half of PLWH reported experiencing stigma due to their status (42\%), while nearly all PLWH had disclosed their HIV status to their friends and family (95\%). Most PLWH (93\%) reported taking ART.

Compared with those without HIV (25\%), more PLWH $(33.9 \%)$ reported experiencing age discrimination, but the difference was not significant $(p=.31)$. Descriptions of negative treatment included being treated as invisible or ignored, body fascism, being viewed as impatient, lacking sexual interest, and employment discrimination. Examples of positive treatment included being offered or given discounts due to age, people thinking they look younger than they are, and more respect or help. Regarding other indicators of successful aging, there were no differences between the two groups in frequency of exercise $(p=.41)$, work or volunteering $(p=.74)$, or feelings of isolation $(p=.26)$. However, among PLWH not working or volunteering was significantly associated with feelings of isolation $(p=.005)$. PLWH reported owning a pet at a higher frequency $(p=.08)$ and also reported significantly lower frequencies of living with another person $(p<.001)$. For people without HIV, there was a significant association between feelings of isolation and not living with someone $(p<.001)$, but this was not true for PLWH. 
FIG. 1. Histograms display the proportion of participants having each number of comorbid conditions across three categories of conditions stratified by HIV status. HIV is included as an infectious disease.

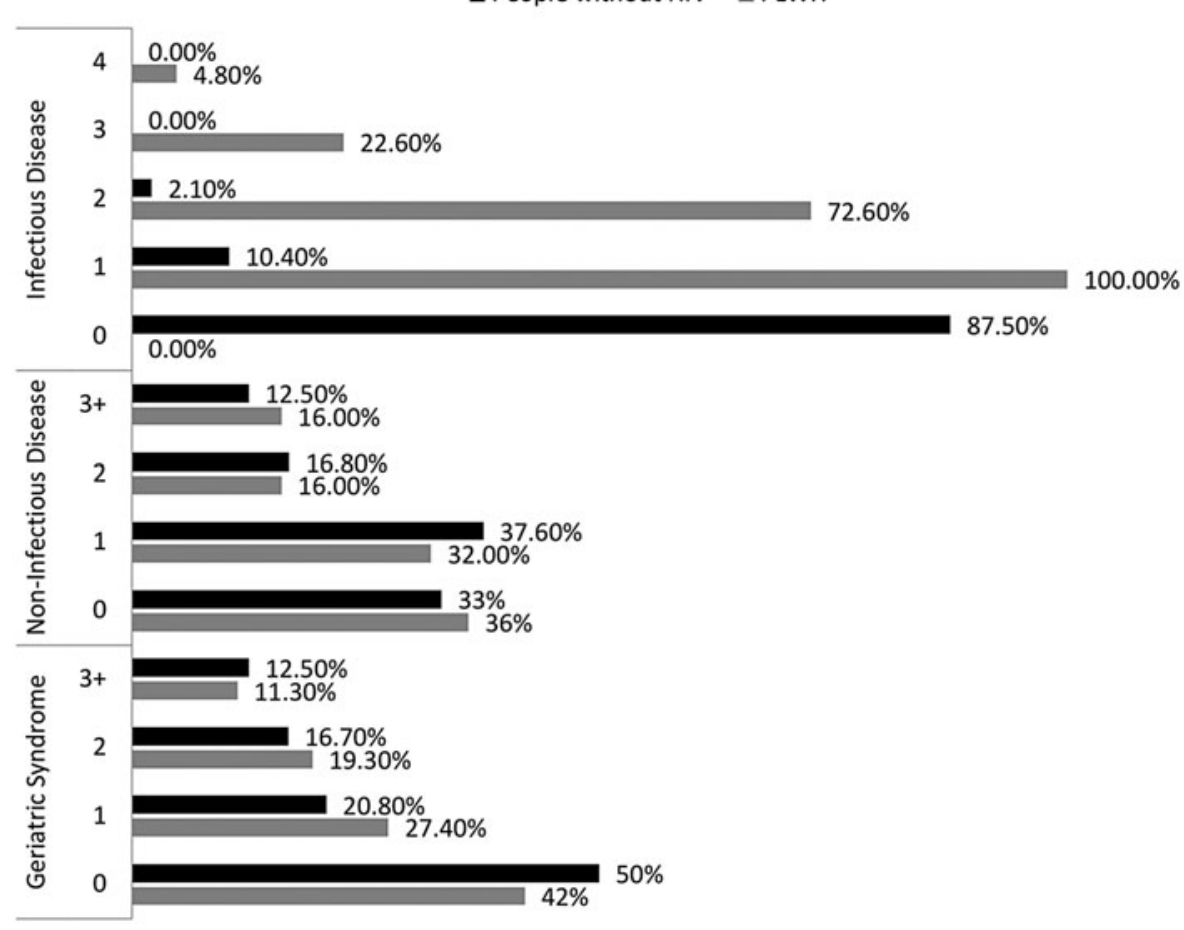

\section{Discussion}

This study sought to assess successful aging among older PLWH compared with older people without HIV. Our findings show that compared with people without HIV, PLWH had significantly higher reports of depression, receiving disability benefits, lower frequency of living with another person, and reported greater importance of work and volunteer opportunities for avoiding feelings of isolation. However, there were no significant differences in positive treatment, age discrimination, experiences of stigma, health rating, pain score, sexual activity, feelings of isolation, or work or volunteering. Thus, despite the challenges associated with aging with HIV (e.g., higher reports of depression, disability benefits, etc.) PLWH appear to be experiencing successful aging to a similar degree compared with their peers without HIV.

The multimorbidity profiles of the PLWH and people without HIV were similar. Top comorbidities among both groups included hypertension, arthritis, and neuropathy, which supports previous research. ${ }^{23}$ However, PLWH were significantly more likely to report depression than people without HIV. This finding supports previous research, which shows that depression is the most common comorbid condition among PLWH, with prevalence rates three times higher than the general population. ${ }^{24}$

We did not find a significant difference between reported feelings of isolation among PLWH and people without HIV. This could be linked to the finding that most PLWH in our study have disclosed their HIV status to their friends and family, which suggests relatively high social support among the study sample. ${ }^{25,26}$ Also, there is a significant support network available for PLWH in Palm Springs, including Let's Kick AIDS Survivor Syndrome and Positive Life. Relatedly, for people without HIV we found a significant relationship between feelings of isolation and not living with someone, but there was no such association among PLWH. This could reflect the success of the support network for PLWH in Palm Springs and suggests the need for similar community-support resources for older adults without HIV.

We also found that lower participation in volunteer and work activities was significantly associated with feelings of isolation among PLWH, but not for those without HIV. Coupled with our findings that PLWH were less likely to live with or be in a relationship with someone, the association between volunteer/work activities and feelings of isolation suggests that older PLWH must rely more on social support beyond that which is provided by family and friends and highlights the importance of community-support resources for this population. ${ }^{16,17}$ These findings are consistent with other research, which suggests that older adults who live alone face higher risk of social isolation, which reduces the likelihood of successful aging. ${ }^{27}$ Where possible, providers for older PLWH should suggest community-support resources to discourage social isolation and help curtail the negative effects that often stem from it, such as depression.

We also found that PLWH were significantly more likely to report receiving disability benefits than people without HIV, which could be an indicator of the deleterious impact of living with HIV, including lack of work resulting in lower income and lower achievement of ADLs, which is negatively associated with health and well-being. ${ }^{15,28}$ Thus, receiving disability benefits suggests a negative impact on successful aging among older PLWH. However, other key findings from this study may help mitigate the negative impact of disability on successful aging among our sample, such as a high frequency of volunteering and low frequency of feelings of isolation.,29 Indeed, qualitative research has shown that psychosocial factors (e.g., community involvement, social ties) are emphasized more than physical factors (e.g., presence of disease and disability) in older adults' perceptions of successful aging. ${ }^{30,31}$ 
This study has several limitations. First, the majority of participants were white, male, college educated, and resided in or near Palm Springs, California, where there may be more HIV disclosure and less HIV stigma compared with other locations. Therefore, these findings may not be generalizable to other racially/ethnically and socioeconomically diverse populations, other genders, or other communities of PLWH. Further, our sample was relatively small and subject to selection bias due to the geographical location of the study and because the sample was recruited from local organizations and support groups, where participants may have been less isolated than peers not associated with these organizations. In addition, the data collected from participants came from self-reported surveys and could not be independently verified. Therefore, estimates of multimorbidity, disability, and other assessments are subjective measures. Likewise, the presence of comorbid conditions may have been underreported due to relying on participants' recall. In addition, comparing successful aging among PLWH and people without HIV is difficult because PLWH might have a very different aging experience than those without HIV. For instance, HIV is itself a comorbid condition and likely has a significant impact on long-term health. Finally, we did not directly measure successful aging. We can only make inferences about successful aging through measures of physiological and psychosocial factors related to aging with HIV, such as physical health and social engagement-some of which are indirect measures themselves (e.g., social isolation). Future work should develop a composite measure to support a construct of successful aging.

Together, our findings suggest that older PLWH experience successful aging to a similar degree as their peers without HIV. However, our findings show that depression and social isolation remain highly salient issues that threaten successful aging and with which PLWH must contend. Therefore, interventions must focus on mitigating these issues to promote successful aging and could do so by building upon sources of resilience among PLWH, such as social and community involvement through work or volunteering. For example, interventions could identify volunteer, work, or other opportunities for community involvement (e.g., support groups, social clubs) in nearby areas, encourage PLWH to take part, and communicate the numerous benefits of community involvement. To promote successful aging and attenuate the unique challenges associated with aging with HIV, our findings also support the case that providers should focus on giving tailored, patient-centered care to this population, such as screening for depression, directing patients to community-support organizations, and making an effort to respond to patients' highest priorities or problems that most immediately threaten successful aging. ${ }^{32}$

\section{Acknowledgments}

We acknowledge Dr. Steve Scheibel for his tireless dedication to the HIV community. We are grateful to the study participants for their time and energy. We would like to thank Jo Gerrard for editorial assistance.

\section{Author Disclosure Statement}

No competing financial interests exist.

\section{Funding Information}

This study was supported in part by a University of California, Riverside seed grant and discretionary funds.

\section{References}

1. Centers for Disease Control and Prevention: Estimated HIV incidence and prevalence in the United States, 2010-2015, 2018.

2. Deeks SG, Lewin SR, Havlir DV: The end of AIDS: HIV infection as a chronic disease. Lancet 2013;382:1525-1533.

3. Harrison KM, Song R, Zhang X: Life expectancy after HIV diagnosis based on national HIV surveillance data from 25 states, United States. J Acquir Immune Defic Syndr 2010; 53:124-130.

4. Greene M, Justice AC, Covinsky KE: Assessment of geriatric syndromes and physical function in people living with HIV. Virulence 2017;8:586-598.

5. Guaraldi G, Zona S, Brothers TD, et al.: Aging with HIV vs. HIV seroconversion at older age: A diverse population with distinct comorbidity profiles. PLoS One 2015;10: $1-11$.

6. Halkitis PN, Krause KD, Vieira DL: Mental health, psychosocial challenges and resilience in older adults living with HIV. Interdiscip Top Gerontol Geriatr 2017;42:187203.

7. Wong C, Gange SJ, Moore RD, et al.: Multimorbidity among persons living with human immunodeficiency virus in the United States. Clin Infect Dis 2018;66:12301238.

8. Kahana E, Kahana B: Successful aging among people with HIV/AIDS. J Clin Epidemiol 2001;54 Suppl 1:S53-S56.

9. Vance DE, McGuinness T, Musgrove K, Orel NA, Fazeli PL: Successful aging and the epidemiology of HIV. Clin Interv Aging 2011;6:181-192.

10. Rowe JW, Kahn RL: Successful aging. Aging (Milano) 1998;10:142-144.

11. Fazeli PL, Montoya JL, McDavid CN, Moore DJ: Older HIV+ and HIV- adults provide similar definitions of successful aging: A mixed-methods examination. Gerontologist 2018 [Epub ahead of print]; DOI: 10.1093/ geront/gny157.

12. Young Y, Frick KD, Phelan EA: Can successful aging and chronic illness coexist in the same individual? A multidimensional concept of successful aging. J Am Med Dir Assoc 2009; 10:87-92.

13. Solomon P, Letts L, O'Brien KK, Nixon S, Baxter L, Gervais N: "I'm still here, I'm still alive": Understanding successful aging in the context of HIV. Int J STD AIDS 2018;29:172-177.

14. Jeste DV, Savla GN, Thompson WK, et al.: Association between older age and more successful aging: Critical role of resilience and depression. Am J Psychiatry 2013;170: 188-196.

15. Nguyen AL, McNeil CJ, Han SD, Rhodes SD: Risk and protective factors for health-related quality of life among persons aging with HIV. AIDS Care 2018;30:518-522.

16. Brennan-Ing M, Seidel L, Karpiak SE: Social support systems and social network characteristics of older adults with HIV. Interdiscip Top Gerontol Geriatr 2017;42:159172.

17. Solomon P, O’Brien K, Wilkins S, Gervais N: Aging with HIV and disability: The role of uncertainty. AIDS Care 2014;26:240-245. 
18. Brown B, Marg L, Cabral A, et al:: Community-driven health priorities for healthy aging with HIV. J Assoc Nurses AIDS Care 2019;30:119-128.

19. Halkitis PN, Kapadia F, Ompad DC, Perez-Figueroa R: Moving toward a holistic conceptual framework for understanding healthy aging among gay men. J Homosex 2015;62:571-587.

20. Emlet CA, Harris L: Giving back is receiving: The role of generativity in successful aging among HIV-positive older adults. J Aging Health 2018 [Epub ahead of print]; DOI: $10.1177 / 0898264318804320$.

21. Moore RC, Moore DJ, Thompson WK, Vahia IV, Grant I, Jeste DV. A case-controlled study of successful aging in older HIV-infected adults. J Clin Psychiatry 2013;74:e417-e423.

22. Gardner AT: Riverside University Health System-Public Health, Epidemiology and Program Evaluation. Epidemiology of HIV/AIDS in Riverside County, 2016. August 2017. www.researchgate.net/publication/319163168_Epidemiology_ of_HIVAIDS_in_Riverside_County_2016

23. Balderson BH, Grothaus L, Harrison RG, McCoy K, Mahoney C, Catz S: Chronic illness burden and quality of life in an aging HIV population. AIDS Care 2013;25:451-458.

24. Rodriguez-Penney AT, Iudicello JE, Riggs PK, et al.: Comorbidities in persons infected with HIV: Increased burden with older age and negative effects on health-related quality of life. AIDS Patient Care STDs 2013;27:5-16.

25. Poindexter CC, Shippy RA: HIV diagnosis disclosure: Stigma management and stigma resistance. J Gerontol Soc Work 2010;53:366-381.

26. Sankar A, Nevedal A, Neufeld S, Berry R, Luborsky M: What do we know about older adults and HIV? A review of social and behavioral literature. AIDS Care 2011;23:11871207.

27. Fredriksen-Goldsen KI, Kim H-J, Barkan SE, Muraco A, Hoy-Ellis CP: Health disparities among lesbian, gay, and bisexual older adults: Results from a population-based study. Am J Public Health 2013;103:1802-1809.

28. Emlet CA, Brennan DJ, Brennenstuhl S, Rueda S, Hart TA, Rourke SB: Protective and risk factors associated with stigma in a population of older adults living with HIV in Ontario, Canada. AIDS Care 2013;25:1330-1339.

29. Kim SH, Park S: A meta-analysis of the correlates of successful aging in older adults. Res Aging 2017;39:657-677.

30. Reichstadt J, Sengupta G, Depp CA, Palinkas LA, Jeste DV: Older adults' perspectives on successful aging: Qualitative interviews. Am J Geriatr Psychiatry 2010;18:567575.

31. Reichstadt J, Depp CA, Palinkas LA, Folsom DP, Jeste DV: Building blocks of successful aging: A focus group study of older adults' perceived contributors to successful aging. Am J Geriatr Psychiatry 2007;15:194-201.

32. Justice A, Braithwaite RS: Lessons learned from the first wave of aging with HIV. AIDS 2012;26(Suppl 1):S11-S18.

Address correspondence to:

Brandon Brown, $M P H, P h D$ School of Medicine

University of California, Riverside 900 University Avenue Riverside, CA 92521

E-mail: brandon.brown@medsch.ucr.edu

\section{Appendix A1. 28-Item Survey}

1. How old were you on your last birthday?

2. Do you feel that people treat you differently because of your age?
a. No

b. Yes

i. Positive treatment (describe)

ii. Negative treatment (describe)

3 . What is your current gender?
a. Male
b. Female
c. Transgender
d. Other (describe)

4. Which of these racial backgrounds best describes you? (you may choose more than one)

a. White/Caucasian (non-Hispanic).

b. White (Hispanic)

c. Black/African American.

d. Asian.

e. Native Hawaiian/Pacific Islander.

American Indian/Alaskan Native g. More than one race.

h. Unknown.

i. Other (please describe below)

5. Are you currently on antibiotics? Yes/No (please circle one)
a. Yes
i. If yes: which kind?

b. No

6. What is your highest level of schooling?
a. Below high school
b. High school
c. Some college
d. 4-year university
e. Graduate school
f. Other (describe)

7. In general, how would you rate your health?
a. Excellent
b. Very good
c. Good
d. Fair
e. Poor

8. On a scale from 0 to 10 (with " 0 " having no pain and " 10 " having unbearable pain), how would you rate your pain level in your normal activities of daily living? (walking, sitting)

(Appendix continues $\rightarrow$ ) 
9. How often do you exercise (days per week)?

10. Are you currently on disability?
a. Yes
b. No

11. Please check if you currently have any of the following conditions
a. Depression
b. Arthritis
c. Hepatitis
d. Neuropathy
e. Hypertension (high blood pressure)
f. Dermatologic problems
g. Herpes
h. Vision loss
i. Diabetes
j. Neurological problems
k. STD (sexually transmitted infections such as gonorrhea, syphilis, and chlamydia)
1. Hearing loss
m. Pneumonia
n. Respiratory problems
o. Heart condition
p. Broken bones
q. Shingles
r. Migraines
s. Cancer
t. Stroke

12. Do you live with another person?
a. Yes
b. No

13. Do you own a pet?
a. Yes
b. No

14. What is your relationship status?
a. Single
b. In a relationship
c. Engaged

d. Married

e. Widowed

15. Are you currently sexually active?

a. Yes

b. No

16. What is the HIV status of your sexual partner?
a. Negative
b. Positive
c. Don't know

17. When was the last time you had sex?

18. Do you currently work or volunteer?

a. Yes (describe)

b. No

19. Do you feel isolated?

a. No

b. Yes (describe)

20. How many times do you meet with family and/or friends in a typical week?

21. What was the result of your last HIV test?

If it was $\mathrm{HIV+}$

22. In what year were you diagnosed?

23. How long after your diagnosis did you start taking HIV drug therapy?

24. Do you feel any stigma due to your HIV status?

25. Have you told your family or friends that you have HIV?

26. Do you take antiretroviral medications for HIV?

If you were HIV-

27. Do you take PrEP?

For all

28. Have you participated in any clinical trials related to HIV prevention or treatment?

a. Yes

b. No 